\title{
A study of the effects of electric field on two-dimensional inviscid nonlinear free surface flows generated by moving disturbances
}

\author{
M. J. Hunt · J.-M. Vanden-Broeck
}

Received: 3 March 2014 / Accepted: 14 November 2014 / Published online: 21 January 2015

(C) The Author(s) 2015. This article is published with open access at Springerlink.com

\begin{abstract}
Two-dimensional free surface flows generated by a moving disturbance are considered. The flows are assumed to be potential. The effects of electric field, gravity and surface tension are included in the dynamic boundary condition. The disturbance is chosen to be a distribution of pressure moving at a constant velocity. Both linear and nonlinear results are presented. For some values of the parameters, the linear theory predicts unbounded displacements of the free surface. It is shown that this nonuniformity is removed by developing a weakly nonlinear theory. There are then solutions which are perturbations of a uniform stream and others which are perturbations of solitary waves with decaying tails.
\end{abstract}

Keywords Electrohydrodynamics $\cdot$ Free surface flows $\cdot$ Surface tension $\cdot$ Solitary waves

\section{Introduction}

There is an extensive literature on nonlinear free surface flows generated by moving disturbances in the absence of electric field (see, for example, Forbes and Schwartz [1], Parau and Vanden-Broeck [2] and Vanden-Broeck [3] for a review). On the other hand, the effects of electric field on periodic and solitary travelling waves have been studied and many new properties have been discovered (see Papageorgiou and Vanden-Broeck [4], Gleeson et al. [5] and the references cited).

In this paper, the combined problem of nonlinear free surface flows generated by a disturbance moving at a constant velocity $U$ in the presence of electric field is investigated. The importance of interfacial electrohydrodynamic phenomena has been reviewed by Melcher and Taylor [6]. Applications can be found in processes such as the electrostatic liquid film radiator (see Griffing et al. [7] and the references therein).

The fluid is assumed to be incompressible and inviscid and the flow to be irrotational. The moving disturbance can be a submerged object, a surface piercing object or a distribution of pressure. The results are qualitatively independent of the type of disturbance (see Hunt [8]). Here we choose a distribution of pressure. We take a frame of reference moving with the disturbance and seek steady solutions. The basic equations are derived in Sect. 2. We start our investigations in Sect. 3, where we construct linear solutions by assuming that the magnitude of the disturbance is 'small'. We show that for some values of the parameters, linear theory predicts unbounded displacements of the

M. J. Hunt · J.-M. Vanden-Broeck ( $\square)$

Department of Mathematics, University College London, London WC1E 6BT, UK

e-mail: j.vanden-broeck@ucl.ac.uk 
free surface. We then demonstrate in Sect. 4 that this nonuniformity is removed by including nonlinearity. This is achieved by developing a weakly nonlinear theory, where the problem is described by a forced fifth-order Korteweg de Vries Benjamin-Ono equation. It is shown that there are solutions which are perturbations of a uniform stream and others which are perturbations of solitary waves with decaying oscillatory tails.

\section{Formulation}

Consider a two-dimensional, inviscid, incompressible and irrotational flow generated by a disturbance moving at a constant velocity $U$. We introduce cartesian coordinates with the vertical axis directed vertically upwards. The acceleration of gravity $g$ is acting in the negative $y$ direction. The flow (region 1 ) is bounded above by the interface $y=\eta(t, x)$ and below by the horizontal bottom $y=-h$ (see Fig. 1).

The fluid motion in region 1 is described by a velocity potential $\varphi(t, x, y)$. Surface tension with coefficient $\sigma$ acts on the interface. We denote the voltage potential by $V$ and choose $V=0$ at $y=-h$. A vertical electric field is imposed by requiring

$V \approx-E_{0} y$ as $y \rightarrow \infty$

where $E_{0}$ is a constant. We assume that the fluid in region 1 is a perfect conductor so that $V=0$ everywhere in it. The region $y>\eta(t, x)$, denoted as region 2 , is occupied by a dielectric having permittivity $\bar{\epsilon}$.

We assume that the disturbance is a given distribution of pressure $P(t, x)$ acting on the interface $y=\eta(t, x)$ and satisfying $P \rightarrow 0$ as $|x| \rightarrow \infty$.

The potentials $\varphi$ and $V$ satisfy the Laplace equations

$$
\begin{array}{ll}
\frac{\partial^{2} \varphi}{\partial x^{2}}+\frac{\partial^{2} \varphi}{\partial y^{2}}=0 & -h<y<\eta(t, x), \\
\frac{\partial^{2} V}{\partial x^{2}}+\frac{\partial^{2} V}{\partial y^{2}}=0 & y>\eta(t, x),
\end{array}
$$

in regions 1 and 2, respectively. The electric field is given by $\mathbf{E}=-\nabla V$.

On the interface $y=\eta(t, x)$, the kinematic boundary condition and the continuity of normal stresses yield

$\frac{\partial \eta}{\partial t}+u \frac{\partial \eta}{\partial x}=v$

and

$$
[\hat{\mathbf{n}} \cdot T \cdot \hat{\mathbf{n}}]_{1}^{2}=\sigma \nabla \cdot \hat{\mathbf{n}},
$$

Fig. 1 Sketch of the flow

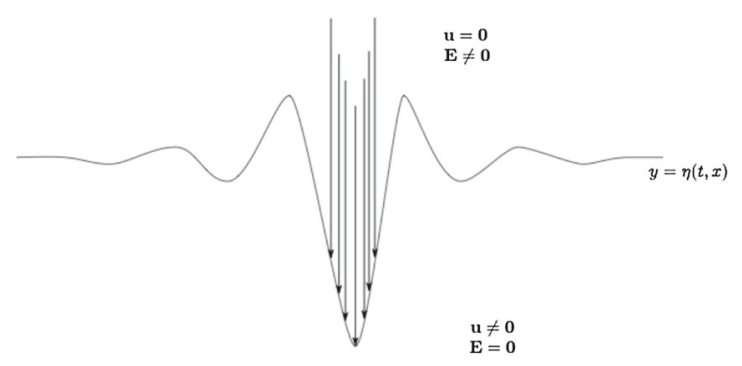


where $[\ldots]_{1}^{2}$ denotes the difference of the values of a quantity across the interface, $\hat{\mathbf{n}}$ is a unit vector in the direction of the normal to the interface and $T$ is the stress tensor defined by

$$
\begin{aligned}
T_{i j} & =-p \delta_{i j}+\Sigma_{i j}, \\
\Sigma_{i j} & =\bar{\epsilon}\left(E_{i} E_{j}-\frac{1}{2} \delta_{i j} E_{k} E_{k}\right) .
\end{aligned}
$$

The quantities $E_{i}$ are the components of the electric field $\mathbf{E}, \Sigma$ is the Maxwell stress tensor, $p$ is the pressure and $\delta_{i j}$ is the Kronecker symbol.

Since $V=0$ in region 1, the components $\Sigma_{i j}$ of the Maxwell stress tensor vanish in region 1 (see (7)). Furthermore, the value $p_{2}$ of the pressure $p$ on the upper part of the interface is equal to the prescribed pressure $P$. Then (5) implies

$p_{1}=P-\frac{1}{1+\left(\frac{\partial \eta}{\partial x}\right)^{2}}\left[\left(\frac{\partial \eta}{\partial x}\right)^{2} \Sigma_{11}-2 \frac{\partial \eta}{\partial x} \Sigma_{12}-\Sigma_{22}\right]-\frac{\sigma \frac{\partial^{2} \eta}{\partial x^{2}}}{\left[1+\left(\frac{\partial \eta}{\partial x}\right)^{2}\right]^{3 / 2}}$,

where

$$
\begin{aligned}
& \Sigma_{11}=\frac{1}{2}\left[\left(\frac{\partial V}{\partial x}\right)^{2}-\left(\frac{\partial V}{\partial y}\right)^{2}\right], \\
& \Sigma_{12}=\frac{\partial V}{\partial x} \frac{\partial V}{\partial y}, \\
& \Sigma_{22}=-\Sigma_{11} .
\end{aligned}
$$

Expression (8) can then be inserted in the Bernoulli equation

$$
\frac{\partial \varphi}{\partial t}+\frac{1}{2}|\nabla \varphi|^{2}+\frac{p}{\rho}+g \eta=C
$$

and evaluated on the lower side of the interface. Here $C$ is the Bernoulli constant. This yields

$$
\partial_{t} \varphi+\frac{1}{2}|\nabla \varphi|^{2}+\frac{P}{\rho}+\frac{1}{\rho} \frac{1}{1+\left(\partial_{x} \eta\right)^{2}}\left(\left(\partial_{x} \eta\right)^{2} \Sigma_{11}-2 \partial_{x} \eta \Sigma_{12}+\Sigma_{22}\right)+g \eta=\frac{\sigma}{\rho} \frac{\partial_{x}^{2} \eta}{\left(1+\left(\partial_{x} \eta\right)^{2}\right)^{3 / 2}}+C .
$$

Since there is no flow through the lower boundary at $y=-h$, we have

$\frac{\partial \phi}{\partial y}=0 \quad$ on $\quad y=-h$.

A further condition comes from the continuity of the tangential component of the electric field across the interface. This implies that the potential $V$ satisfies

$$
\frac{\partial \eta}{\partial x} \frac{\partial V}{\partial y}+\frac{\partial V}{\partial x}=0 \quad \text { or } \quad V=0 \quad \text { on } \quad y=\eta(t, x) .
$$

This completes the formulation of the problem.

Next we introduce the dimensionless variables $\tilde{x}, \tilde{y}, \ldots$ by the relations

$x=h \tilde{x}, \quad y=h \tilde{y}, \quad \eta=h \tilde{\eta}, \quad t=\sqrt{\frac{\rho h^{3}}{\sigma}} \tilde{t}, \quad \varphi=\sqrt{\frac{\sigma h}{\rho} \tilde{\varphi}}, \quad V=E_{0} L \tilde{V}, \quad P=\frac{\sigma}{h} \tilde{p}$.

The dynamic boundary condition (13) becomes

$$
\partial_{\tilde{t}} \tilde{\varphi}+\frac{1}{2}\left(\left(\partial_{\tilde{x}} \tilde{\varphi}\right)^{2}+\left(\partial_{\tilde{y}} \tilde{\varphi}\right)^{2}\right)+Q_{1}+\tilde{p}+\tau^{-1} \tilde{\eta}=\frac{\partial_{\tilde{x}}^{2} \tilde{\eta}}{\left(1+\left(\partial_{\tilde{x}} \tilde{\varphi}\right)^{2}\right)^{3 / 2}}+K,
$$


where

$E_{\mathrm{b}}=\frac{\bar{\epsilon} E_{0}^{2} h}{\sigma}$ and $\tau=\frac{\sigma}{\rho g h^{2}}$.

Here

$Q_{1}=\frac{E_{\mathrm{b}}}{1+\left(\partial_{\tilde{x}} \tilde{\eta}\right)^{2}}\left[\left(\partial_{\tilde{x}} \tilde{\eta}\right)^{2} \tilde{\Sigma}_{11}-2 \partial_{\tilde{x}} \tilde{\eta} \tilde{\Sigma}_{12}+\tilde{\Sigma}_{22}\right]$.

Equation (1) gives

$\frac{\partial \tilde{V}}{\partial \tilde{y}} \rightarrow-1$ as $\tilde{y} \rightarrow \infty$.

Equations (2), (3), (4), (14) and (15) remain unchanged in dimensionless variables.

The distribution of pressure is moving at a constant velocity $U$. We choose a frame of reference moving with the pressure distribution and seek steady solutions. Therefore, the partial derivatives with respect to $\tilde{t}$ in (4) and (17) can be dropped.

\section{Linear theory}

When $\tilde{p}=0$, the set of equations derived in the previous section has the exact trivial solution

$\tilde{\eta}=0, \tilde{\varphi}=U \tilde{x} \quad$ and $\quad \tilde{V}=-\tilde{y}$.

We seek a solution which is a perturbation of this exact solution by writing

$\tilde{p}=\epsilon p_{1}$,

$\tilde{\eta}=\epsilon \eta_{1}$,

$\tilde{V}=-y+\epsilon V_{1}$,

$\tilde{\varphi}=U x+\epsilon \varphi_{1}$.

Here $\epsilon$ is a small positive parameter.

Substituting (21)-(24) into the dimensionless equations and dropping the tildes and the terms of order $\epsilon^{2}$ and higher give the linear system

$$
\begin{aligned}
& \frac{\partial^{2} \varphi_{1}}{\partial x^{2}}+\frac{\partial^{2} \varphi_{1}}{\partial y^{2}}=0 \text { in }-1<y<0, \\
& \frac{\partial^{2} V_{1}}{\partial x^{2}}+\frac{\partial^{2} V_{1}}{\partial y^{2}}=0 \text { in } y>0, \\
& U \frac{\partial \eta_{1}}{\partial x}=\frac{\partial \varphi_{1}}{\partial y} \text { on } y=0, \\
& U \frac{\partial \varphi_{1}}{\partial x}+\tau^{-1} \eta_{1}+p_{1}(x)+E_{\mathrm{b}} \frac{\partial V_{1}}{\partial y}=\frac{\partial^{2} \eta_{1}}{\partial x^{2}}+\frac{E_{\mathrm{b}}}{2} \text { on } y=0, \\
& -\frac{\partial \eta_{1}}{\partial x}+\frac{\partial V_{1}}{\partial x}=0 \text { on } y=0, \\
& \frac{\partial \varphi_{1}}{\partial y}=0 \text { on } y=-1, \\
& \frac{\partial V_{1}}{\partial y} \rightarrow 0 \text { as } y \rightarrow \infty .
\end{aligned}
$$

This system can easily be solved by Fourier transforms. The solutions are

$\varphi_{1}=\int_{\mathbb{R}} A(k) \mathrm{e}^{\mathrm{i} k x} \cosh k(y+1) \mathrm{d} k$, 


$$
\begin{aligned}
& V_{1}=\int_{\mathbb{R}}-\frac{\mathrm{i}}{U} A(k) \mathrm{e}^{-|k| y} \mathrm{e}^{\mathrm{i} k x} \sinh k \mathrm{~d} k, \\
& \eta_{1}=\int_{\mathbb{R}}-\frac{\mathrm{i}}{U} A(k) \mathrm{e}^{\mathrm{i} k x} \sinh k \mathrm{~d} k,
\end{aligned}
$$

where

$$
A(k)=\frac{-\mathrm{i} E(k)}{-k U \cosh k+\left((\tau U)^{-1}-E_{\mathrm{b}} U^{-1}|k|+k^{2} U^{-1}\right) \sinh k},
$$

and $E(k)$ is the Fourier transform of the moving pressure distribution $p_{1}(x)$, i.e.

$p_{1}(x)=\int_{\mathbb{R}} E(k) \mathrm{e}^{\mathrm{i} k x} \mathrm{~d} k$.

We shall present results for the particular choice

$p_{1}(x)=\frac{1}{2} \mathrm{e}^{-x^{2}}$

for which

$E(k)=\frac{1}{2} \sqrt{\pi} \mathrm{e}^{-k^{2} / 4}$.

Since $E(k)$ in (38) is an even function of $k$, we can use (35) to rewrite (34) as

$\eta_{1}=\int_{0}^{\infty} \frac{-E(k) \sinh k \cos k x}{-k U^{2} \cosh k+\left(\tau^{-1}-E_{\mathrm{b}} k+k^{2}\right) \sinh k} \mathrm{~d} k$.

In terms of the dimensional variables, (39) can be rewritten as

$$
\frac{\eta_{1}(x)}{h}=-\tau \int_{0}^{\infty} \frac{E(k h) \tanh k h \cos k x}{-k h F^{2}+\left(1-E_{\mathrm{b}} \tau k h+k^{2} h^{2} \tau\right) \tanh k h} \mathrm{~d}(k h) .
$$

Some insight into the solutions can be gained by looking at the dispersion relation of a train of waves travelling on the free surface. Then we set $p_{1}=0$ in (28). A solution of (25)-(27), (29)-(31) is then

$\varphi_{1}=D \cos k x \cosh k(y+1)$,

$V_{1}=\frac{1}{U} D \sin k x \mathrm{e}^{-|k| y} \sinh k$,

$\eta_{1}=\frac{1}{U} D \sin k x \sinh k$,

where $D$ is a constant. The solution (41)-(42) corresponds to a train of waves of wavenumber $k$ and phase speed $U$ when viewed in a frame of reference moving with the wave. Substituting (41)-(43) into (28) yields the dispersion relation

$U^{2}=\left(\frac{1}{\tau k}-E_{\mathrm{b}} \frac{|k|}{k}+k\right) \tanh k$.

The relation (44) can be rewritten in terms of the dimensional variables as

$F^{2}=\left(\frac{1}{k h}-E_{\mathrm{b}} \tau \frac{|k h|}{k h}+\tau k h\right) \tanh k h$, 


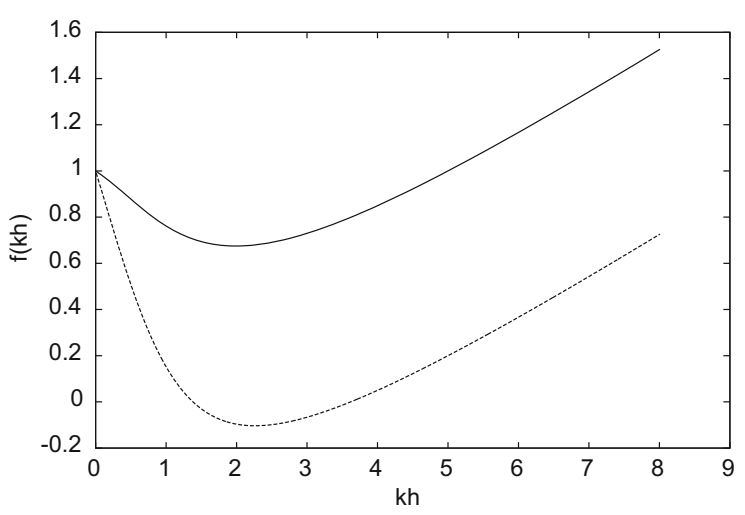

Fig. 2 Values of $f(k h)$ versus $k h$ for $\tau=0.2$. The solid curve corresponds to $E_{\mathrm{b}}=1$. Since $E_{\mathrm{b}}^{2}<4 / \tau$, the minimum value of $f(k h)$ is positive. The broken curve corresponds to $E_{\mathrm{b}}=5$. Since $E_{\mathrm{b}}^{2}>4 / \tau$, the minimum value of $f(k h)$ is negative

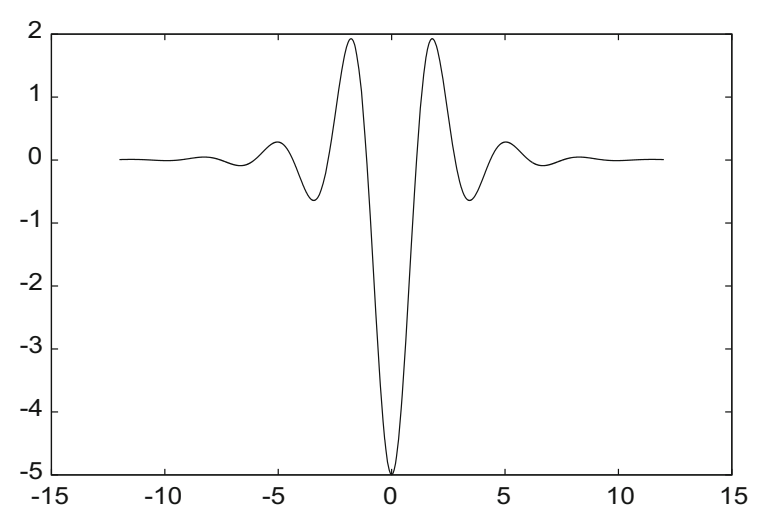

Fig. 3 Free surface profile for $E_{\mathrm{b}}=1, \tau=0.2$ and $F^{2}=0.65$

where $F$ is the Froude number defined by

$F=\frac{U}{\sqrt{g h}}$.

It can be seen that the denominator of the integrand in (40) vanishes when there are real values of $k h$ satisfying (45). In this paper, we choose values of $\tau$ and $E_{\mathrm{b}}$ so that this does not occur. To show that this is possible, we denote by $f(k h)$ the right-hand side of (45) and assume $k h>0$ (similar results hold for $k h<0$ since $f(k h)$ is an even function of $k h$ ). Therefore

$f(k h)=\left(\frac{1}{k h}-E_{\mathrm{b}} \tau+\tau k h\right) \tanh k h$.

Next we note that

$f(k h) \approx 1-E_{\mathrm{b}} \tau k h$ as $k h \rightarrow 0$

and

$f(k h) \rightarrow \infty$ as $k h \rightarrow \infty$.

Relations (48) and (49) show that $f(k h)$ is a decreasing function of $k h$ for $k h$ small and an increasing function of $k h$ for $k h$ large. It follows that $f(k h)$ must have a minimum (say $\delta$ ) for some value $k h=(k h)^{*}$. The value of $\delta$ will be positive if $E_{\mathrm{b}}^{2}<4 / \tau$. For, if $\delta<0$, there must be by continuity real values of $k h$ for which $f(k h)=0$. This will occur if the bracket on the right-hand side of (47) vanishes for some values of $k h$. This gives a quadratic equation which has real roots when $E_{\mathrm{b}}^{2}>4 / \tau$. Therefore, $\delta>0$ when $E_{\mathrm{b}}^{2}<4 / \tau$. Assuming this, we see that there will be no real $k$ satisfying (44) if $F^{2}<\delta$.

In summary we assume

$E_{\mathrm{b}}^{2}<\frac{4}{\tau}$ and $F<\sqrt{\delta}$

The properties of $f(k h)$ are illustrated in Fig. 2.

When (50) is satisfied, there are no linear waves travelling at the velocity $U$ and the free surface is flat in the far field. Solutions when (50) is not satisfied are discussed in [8]. 
Fig. 4 Values of $\left|\eta_{1}(0)\right|$ versus $F^{2}$ for $\tau=0.2$ and $E_{\mathrm{b}}=1$

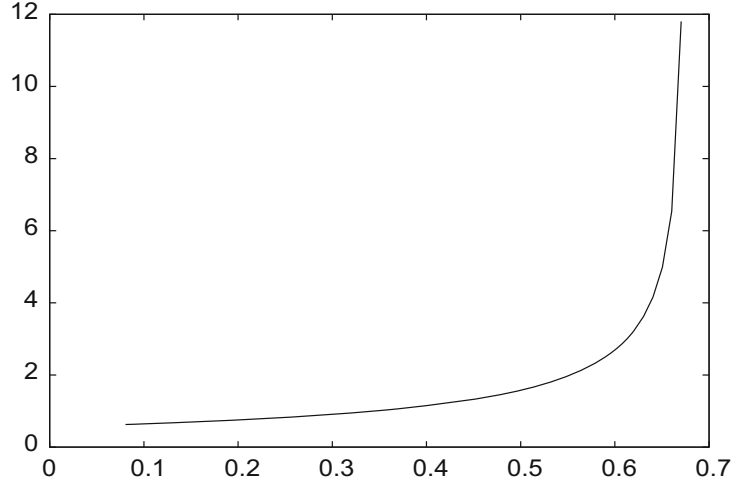

A typical free surface profile is shown in Fig. 3. It was obtained by integrating numerically (39) with $E(k)$ defined by (38). Since $\delta \approx 0.67$, the conditions (50) are satisfied. The profile is characterised by decaying oscillations as $|x| \rightarrow \infty$.

In Fig. 4, we present the values of the maximum displacement $\left|\eta_{1}(0)\right|$ of the free surface versus $F^{2}$. We chose $\tau=0.2$ and $E_{\mathrm{b}}=1$ so that $\delta \approx 0.675$. The curve of Fig. 4 has a vertical asymptote at $F^{2}=\delta$. This follows analytically by noting that the denominator of the integrand in (40) is different from zero when $F^{2}<\delta$ but tends to zero as $F^{2} \rightarrow \delta$. This implies that the linear theory (which assumes that $\epsilon \eta_{1}$ is small when $\epsilon$ is small) becomes invalid as $F^{2} \rightarrow \delta$. This is because for any $\epsilon>0$ (however small), $\epsilon \eta_{1}$ can be made arbitrary large by taking $F^{2}$ sufficiently close to $\delta$. We show in the next section how to remove this nonuniformity by introducing some nonlinearity in the formulation.

\section{Weakly nonlinear theory}

Given a typical velocity $c_{0}=\sqrt{g h}$, a typical amplitude $a$ and a typical length scale $L$, we define the following dimensionless variables:

$$
\begin{aligned}
& x=L \hat{x}, \quad t=\frac{L}{c_{0}} \hat{t}, \quad \eta=a \hat{\eta}, \quad \varphi=\frac{g L a}{c_{0}} \hat{\varphi}, \quad y^{(1)}=h \hat{y}, \quad y^{(2)}=L \hat{Y}, \quad V=L E_{0} \hat{V}, \\
& P=\rho g a \hat{P},
\end{aligned}
$$

and the parameters

$\alpha=\frac{a}{h}, \quad \beta=\frac{h^{2}}{L^{2}}$.

Here $y^{(1,2)}$ denote the $y$-coordinates in the regions 1 and 2, respectively. In terms of the variables (51), the governing equations become

$$
\begin{aligned}
& \frac{\partial^{2} \hat{\varphi}}{\partial \hat{x}^{2}}+\frac{1}{\beta} \frac{\partial^{2} \hat{\varphi}}{\partial \hat{y}^{2}}=0 \quad-1<\hat{y}<\alpha \hat{\eta}, \\
& \frac{\partial^{2} \hat{V}}{\partial \hat{x}^{2}}+\frac{\partial^{2} \hat{V}}{\partial \hat{Y}^{2}}=0 \quad Y>\sqrt{\beta} \alpha \hat{\eta}, \\
& \frac{\partial \hat{\eta}}{\partial \hat{t}}+\alpha \frac{\partial \hat{\varphi}}{\partial \hat{x}} \frac{\partial \hat{\eta}}{\partial \hat{x}}=\frac{1}{\beta} \frac{\partial \hat{\varphi}}{\partial \hat{y}} \text { on } \hat{y}=\alpha \hat{\eta}, \\
& \frac{\beta \tau \partial_{\hat{x}}^{2} \hat{\eta}}{\left(1+\alpha^{2} \beta\left(\partial_{\hat{x}} \hat{\eta}\right)^{2}\right)^{3 / 2}}+C=\frac{\partial \hat{\varphi}}{\partial \hat{t}} \frac{1}{2}\left[\alpha\left(\frac{\partial \hat{\varphi}}{\partial \hat{x}}\right)^{2}+\frac{\alpha}{\beta}\left(\frac{\partial \hat{\varphi}}{\partial \hat{y}}\right)^{2}\right]+\hat{P}+\hat{\eta}+\frac{A}{\alpha} \frac{1}{1+\alpha^{2} \beta\left(\partial_{\hat{x}} \hat{\eta}\right)^{2}} \\
& \times\left[\frac{1}{2}\left(\alpha^{2} \beta\left(\partial_{\hat{x}} \hat{\eta}\right)^{2}-1\right)\left[-\left(\frac{\partial \hat{V}}{\partial \hat{x}}\right)^{2}+\left(\frac{\partial \hat{V}}{\partial \hat{Y}}\right)^{2}\right]+2 \alpha \beta^{1 / 2} \partial_{\hat{x}} \hat{\eta} \frac{\partial \hat{V}}{\partial \hat{x}} \frac{\partial \hat{V}}{\partial \hat{Y}}\right],
\end{aligned}
$$


$\frac{\partial \hat{V}}{\partial \hat{x}}+\alpha \sqrt{\beta} \frac{\partial \hat{\eta}}{\partial \hat{x}} \frac{\partial \hat{V}}{\partial \hat{Y}}=0 \quad$ on $\quad Y=\sqrt{\beta} \alpha \hat{\eta}$,

$\varphi_{\hat{y}}=0$ on $\hat{y}=-1$,

$\hat{V}_{\hat{Y}} \rightarrow 1$ as $\hat{Y} \rightarrow \infty$,

where $C$ is a constant. The dimensionless number $A$ in (56) is defined by

$A=\frac{\bar{\epsilon} E_{0}^{2}}{\rho g h}$.

Using (18) we note that

$A=E_{\mathrm{b}} \tau$.

We now introduce the canonical Korteweg de Vries (KdV) scaling

$\alpha=\beta=\varepsilon \ll 1$

and use the change of coordinates

$T=\varepsilon \hat{t}, \quad X=\hat{x}-\hat{t}$.

Next we substitute (62) and (63) into (53)-(59) and expand in powers of $\epsilon$ by assuming

$\varphi(T, X, y)=\varphi_{0}(T, X, y)+\varepsilon \varphi_{1}(T, X, y)+\cdots$,

$V(T, X, Y)=Y+\varepsilon^{3 / 2} V_{1}(T, X, Y)+\cdots$,

$\eta(T, X)=\eta_{0}(T, X)+\varepsilon \eta_{1}(T, X)+\cdots$

Here (and in the remaining part of this calculation) we drop the hats. We write the pressure $P$ in (56) as

$P=\epsilon^{n} p$,

where $n$ is an integer to be determined. The asymptotic expansion of $V_{1}$ deserves an explanation. Writing the expansion for $V_{1}$ as $V=Y+\delta(\varepsilon) V_{1}$ and inserting it into (57) give

$\delta(\varepsilon) \frac{\partial V_{1}}{\partial X}+\varepsilon^{3 / 2} \frac{\partial \eta}{\partial X}\left(1+\delta(\varepsilon) \frac{\partial V_{1}}{\partial Y}\right)=0$,

which shows that $\delta(\varepsilon)=\varepsilon^{3 / 2}$. The condition (57) becomes to leading order

$\frac{\partial V_{1}}{\partial X}+\frac{\partial \eta_{0}}{\partial X}=0 \quad$ on $Y=0$.

The leading order terms for the unsteady Bernoulli equation (56) are

$\frac{A}{\varepsilon}\left[-\varepsilon^{3 / 2} \frac{\partial V_{1}}{\partial Y}\right]+\epsilon^{n} p(X)$.

From (69), we see that the electric field contributes to the order $\epsilon$ terms in (56) when $A \approx \epsilon^{1 / 2}$. Hence we write

$A=\epsilon^{1 / 2} \bar{A}$.

Similarly, the distribution of pressure contributes to the order $\epsilon$ terms in (56) if $n=1$ in (67).

The quantity $\partial V_{1} / \partial Y$ in (69) must be determined by solving Laplace's equation subject to (68) and the condition $V_{1} \rightarrow 0$ as $Y \rightarrow \infty$ that follows from (59). Using the Cauchy integral formula and the analysis in [4], we obtain

$\frac{\partial V_{1}}{\partial Y}(T, X, 0)=\mathcal{H}\left[\frac{\partial V_{1}}{\partial X}(T, X, 0)\right]=-\mathcal{H}\left[\frac{\partial \eta_{0}}{\partial X}\right]$,

where $\mathcal{H}$ denotes the Hilbert transform operator defined by

$\mathcal{H}[g]=\frac{1}{\pi} \mathrm{PV} \int_{-\infty}^{\infty} \frac{g(\zeta)}{\zeta-x} \mathrm{~d} \zeta$ 
with PV denoting the Cauchy principal value form.

Equations (53), (56) and (58) give at $O\left(\epsilon^{0}\right)$

$\varphi_{0}=\varphi_{0}(T, X), \quad \eta_{0}=\frac{\partial \varphi_{0}}{\partial X}(T, X)$.

Similarly, we obtain at the order $O(\epsilon)$

$\varphi_{1}=-\frac{1}{2} y^{2} \frac{\partial^{2} \varphi_{0}(T, X)}{\partial X^{2}}+\psi_{1}(T, X)$,

$\eta_{1}=-\frac{\partial \varphi_{0}}{\partial T}-\frac{1}{2}\left[\frac{\partial \varphi_{0}}{\partial X}\right]^{2}+\left(-\frac{1}{2}+\tau\right) \frac{\partial^{3} \varphi_{0}}{\partial X^{3}}+\frac{\partial \psi_{1}}{\partial X}-p+\bar{A} \mathcal{H}\left[\frac{\partial \eta_{0}}{\partial X}\right]$,

where the function $\psi_{1}$ is to be determined.

The relations (73), (74) and (75) provide three equations for the four unknowns $\eta_{0}, \eta_{1}, \varphi_{1}$ and $\psi_{1}$. To complete the calculation, we go to the order $O\left(\epsilon^{2}\right)$ to obtain the system

$\frac{\partial^{2} \varphi_{1}}{\partial X^{2}}+\frac{\partial^{2} \varphi_{2}}{\partial y^{2}}=0-1<y<0$,

$\frac{\partial \eta_{0}}{\partial T}-\frac{\partial \eta_{1}}{\partial X}+\frac{\partial \varphi_{0}}{\partial X} \frac{\partial \eta_{0}}{\partial X}-\frac{\partial \varphi_{2}}{\partial y}-\eta_{0} \frac{\partial^{2} \varphi_{1}}{\partial y^{2}}-\eta_{1} \frac{\partial^{2} \varphi_{0}}{\partial y^{2}}-\frac{\eta_{0}^{2}}{2} \frac{\partial^{3} \varphi_{0}}{\partial y^{3}}=0 \quad$ on $\quad y=0$,

$\frac{\partial \varphi_{2}}{\partial y}=0$ on $y=-1$.

We have here omitted the contribution of order $\epsilon^{2}$ from (56) which is not needed. Solving (76) gives

$\frac{\partial \varphi_{2}}{\partial y}(y=1)=\frac{1}{6} \frac{\partial^{4} \varphi_{0}}{\partial X^{4}}-\frac{\partial^{2} \psi_{1}}{\partial X^{2}}$,

which is substituted in (77). The final equation for $\eta_{0}$ is then obtained by eliminating all variables in favour of $\eta_{0}$ using (73)-(75). This yields

$2 \frac{\partial \eta_{0}}{\partial T}+3 \eta_{0} \frac{\partial \eta_{0}}{\partial X}+\left(\frac{1}{3}-\tau\right) \frac{\partial^{3} \eta_{0}}{\partial X^{3}}+\bar{A} \mathcal{H}\left[\frac{\partial^{2} \eta_{0}}{\partial X^{2}}\right]+\frac{\mathrm{d} p}{\mathrm{~d} X}=0$.

We can rewrite (80) in terms of the dimensional variables as

$\frac{\partial \eta}{\partial t}+c_{0} \frac{\partial \eta}{\partial x}+\frac{3}{2} \frac{c_{0}}{h} \eta \frac{\partial \eta}{\partial x}+\left(\frac{1}{3}-\tau\right) \frac{h^{2} c_{0}}{2} \frac{\partial^{3} \eta}{\partial x^{3}}+\frac{E_{\mathrm{b}} \tau c_{0} h}{2} \frac{\partial^{2}}{\partial x^{2}} \mathcal{H}(\eta)+\frac{c_{0}}{2 \rho g} p^{\prime}(x)=0$.

Equation (81) is a weakly nonlinear model for the problem formulated in Sect. 2. It reduces to a forced Korteweg de Vries equation when $E_{\mathrm{b}}=0$. For $E_{\mathrm{b}} \neq 0$, it can be viewed as a forced Benjamin-Ono Korteweg de Vries equation. It generalises the unforced equation derived in [5].

We note that the third term in (81) vanishes when $\tau=1 / 3$. A new weakly nonlinear equation can be derived by assuming that $\tau$ is close to $1 / 3$. The scaling defined by (62) and (63) is first replaced by

$\alpha=\epsilon^{2}, \quad \beta=\epsilon$,

$T=\epsilon^{2} \hat{t}, \quad X=\hat{x}-\hat{t}$.

As before we expand in powers of $\epsilon$ by writing

$\eta=\eta_{0}+\epsilon \eta_{1}+\epsilon^{2} \eta_{2}+\cdots$,

$\varphi=\varphi_{0}+\epsilon \varphi_{1}+\epsilon^{2} \varphi_{2}+\cdots$,

$V=Y+\epsilon^{5 / 2} V_{1}+\cdots$ 
The scaling in (86) follows from (69) by noting that the power $3 / 2$ in (4) is now replaced by $5 / 2$. In addition, we introduce the detuning parameters

$\tau=\frac{1}{3}+\epsilon \tau_{1}+\epsilon^{2} \tau_{2}+\cdots, \quad A=\epsilon^{3 / 2} A^{\star}$.

We also choose $n=2$ in (67). This choice and the scaling for $A$ enable the pressure distribution and the electric field to compete with the third- and fifth-order dispersion terms of the usual $\mathrm{KdV}$ analysis. This happens now at $O\left(\epsilon^{2}\right)$ in the Bernoulli equation. The analysis proceeds as in the previous case, but we need now to go to $O\left(\epsilon^{3}\right)$. The leading order terms are still given by (73). Using these solutions, we obtain at the order $O(\epsilon)$

$\varphi_{1}=-\frac{y^{2}}{2} \frac{\partial^{2} \varphi_{0}}{\partial X^{2}}+\bar{\psi}_{1}(T, X)$,

$\eta_{1}=-\frac{1}{6} \frac{\partial^{3} \varphi_{0}}{\partial X^{3}}+\frac{\partial \bar{\psi}_{1}}{\partial X}$

where $\bar{\psi}_{1}$ is to be found.

At the order $\epsilon^{2}$, we obtain

$$
\begin{aligned}
& \varphi_{2}=\frac{y^{4}}{24} \frac{\partial^{4} \varphi_{0}}{\partial X^{4}}-\frac{y^{2}}{2} \frac{\partial^{2} \bar{\psi}_{1}}{\partial X^{2}}+\bar{\psi}_{2}(T, X), \\
& \frac{\partial \varphi_{0}}{\partial T}-\frac{\partial \varphi_{2}}{\partial X}+\frac{1}{2}\left[\frac{\partial^{2} \varphi_{0}}{\partial X^{2}}\right]^{2}+\eta_{2}+P-\tau_{1} \frac{\partial^{2} \eta_{0}}{\partial X^{2}}-\frac{1}{3} \frac{\partial^{2} \eta_{1}}{\partial X^{2}}+A^{*} \mathcal{H}\left[\frac{\partial^{2} \eta_{0}}{\partial X^{2}}\right]=0 \text { on } y=0 .
\end{aligned}
$$

Solving at the order $\epsilon^{3}$, we obtain

$$
\begin{aligned}
& \frac{\partial \varphi_{3}}{\partial y}=-\frac{y^{5}}{120} \frac{\partial^{6} \varphi_{0}}{\partial X^{6}}+\frac{y^{3}}{6} \frac{\partial^{3} \bar{\psi}_{2}}{\partial X^{3}}-y \frac{\partial^{2} \bar{\psi}_{2}}{\partial X^{2}}, \\
& \frac{\partial \eta_{2}}{\partial X}=\frac{\partial \eta_{0}}{\partial T}+\frac{\partial \varphi_{0}}{\partial X} \frac{\partial \eta_{0}}{\partial X}-\frac{\partial \varphi_{3}}{\partial y}-\eta_{0} \frac{\partial^{2} \varphi_{1}}{\partial y^{2}} \text { on } y=0 .
\end{aligned}
$$

Next we use (73) and substitute (93) into (93) to express $\partial \eta_{2} / \partial X$ in terms of $\eta_{0}, \bar{\psi}_{1}$ and $\bar{\psi}_{2}$. Finally differentiating (91) with respect to $X$ and eliminating $\partial \eta_{2} / \partial X$ and $\partial \eta_{1} / \partial X$ yield the equation

$2 \frac{\partial \eta_{0}}{\partial T}+\frac{1}{45} \frac{\partial^{5} \eta_{0}}{\partial X^{5}}+3 \eta_{0} \frac{\partial \eta_{0}}{\partial X}-\tau_{1} \frac{\partial^{3} \eta_{0}}{\partial X^{3}}+A^{*} \mathcal{H}\left[\frac{\partial^{2} \eta_{0}}{\partial X^{2}}\right]+\frac{\mathrm{d} P}{\mathrm{~d} X}=0$.

In terms of the original variables, the equation is

$$
\partial_{t} \eta+c_{0} \partial_{x} \eta+\frac{1}{90} c_{0} h^{4} \partial_{x}^{5} \eta+\frac{3}{2} \frac{c_{0}}{h} \eta \partial_{x} \eta-\frac{1}{2}\left(\tau-\frac{1}{3}\right) c_{0} h^{2} \partial_{x}^{3} \eta+\frac{1}{2} E_{\mathrm{b}} \tau h c_{0} \partial_{x}^{2} \mathcal{H}[\eta]+c_{0} \frac{p^{\prime}}{2 \rho g}=0 .
$$

Equation (95) is another weakly nonlinear model of the problem formulated in Sect. 2. It was derived by assuming shallow water (i.e. small $\beta$ ), small free surface disturbance (i.e. small $\alpha$ ), small values of $E_{\mathrm{b}}$ and values of $\tau$ close to $1 / 3$.

There is a linearised version of (95) which is obtained by dropping the nonlinear term (i.e. the fourth term in (95)).

The solutions of linearised version of Eq. (95) agree with those obtained in Sect. 3 in the limit $k h \rightarrow 0$. To check this, we assume $p=0$ and we derive the linear dispersion relation associated with the linearised version of (95) by seeking a solution of the linearised version of (95) of the form

$\eta=a \cos (k x-\omega t)$. 
Substituting (96) into the linearised version of (95) gives

$\omega=c_{0} k+\frac{1}{90} c_{0} h^{4} k^{5}+\frac{1}{2}\left(\tau-\frac{1}{3}\right) c_{0} h^{2} k^{3}-\frac{E_{\mathrm{b}} \tau h k^{2} c_{0} \operatorname{sgn}(k)}{2}$.

Since $\omega=U k$, we can rewrite (97) as

$$
F=\frac{U}{c_{0}}=1+\frac{1}{90}(h k)^{4}+\frac{1}{2}\left(\tau-\frac{1}{3}\right)(h k)^{2}-\frac{E_{\mathrm{b}} \tau h k \operatorname{sgn}(k)}{2},
$$

where $F$ is the Froude number defined by (46). To compare with (45), we first square (98). This yields

$$
F^{2}=1+\left(\tau-\frac{1}{3}\right)(k h)^{2}+\frac{1}{45}(k h)^{4}-E_{\mathrm{b}} \tau k h \operatorname{sgn}(k)+\cdots
$$

On the other hand, expanding (45) for $k h$ small gives

$$
F^{2}=1+\left(\tau-\frac{1}{3}\right)(k h)^{2}+\left(\frac{2}{15}-\frac{\tau}{3}\right)(k h)^{4}-E_{\mathrm{b}} \tau k h \operatorname{sgn}(k)+\cdots
$$

Since the linearised version of (95) was derived for $\tau$ close to $1 / 3$, we can replace the third term in (100) by $1 / 45$. Equation (100) reduces then to (99).

A graph of the values of $F$ predicted by (98) versus $k h$ for $E_{\mathrm{b}}=2$ and $\tau=0.1$ is presented in Fig. 5. There is a minimum value $F_{\min } \approx 0.455$ of $F$. This finding is similar to the results found in Sect. 3 for the full linear problem (see Fig. 2).

As in Sect. 3, we assume that the distribution of pressure moves with a constant velocity $U$. We choose a frame of reference moving with the pressure distribution and seek steady solutions. This is achieved by substituting $\eta=f(x-U t)$ in (95). This yields

$$
-U \partial_{x} f+c_{0} \partial_{x} f+\frac{1}{90} c_{0} h^{4} \partial_{x}^{5} f+\frac{3}{2} \frac{c_{0}}{h} f \partial_{x} f-\frac{c_{0}}{2}\left(\tau-\frac{1}{3}\right) h^{2} \partial_{x}^{3} f+\frac{1}{2} E_{\mathrm{b}} \tau h c_{0} \partial_{x}^{2} \mathcal{H}[f]+c_{0} \frac{p^{\prime}}{2 \rho g}=0 .
$$

Similarly, the linearised version of (95) gives for the linearised version

$$
-U \partial_{x} f+c_{0} \partial_{x} f+\frac{1}{90} c_{0} h^{4} \partial_{x}^{5} f-\frac{c_{0}}{2}\left(\tau-\frac{1}{3}\right) h^{2} \partial_{x}^{3} f+\frac{1}{2} E_{\mathrm{b}} \tau h c_{0} \partial_{x}^{2} \mathcal{H}[f]+c_{0} \frac{p^{\prime}}{2 \rho g}=0 .
$$

Equations (101) and (102) can be immediately integrated with respect to $x$ to yield

$$
(1-F) f+\frac{1}{90} h^{4} \partial_{x}^{4} f+\frac{3}{4} f^{2}-\frac{1}{2}\left(\tau-\frac{1}{3}\right) h^{2} \partial_{x} f+\frac{1}{2} E_{\mathrm{b}} \tau h \partial_{x} \mathcal{H}[f]+\frac{p}{2 \rho g}=0
$$

and

$$
(1-F) f+\frac{1}{90} h^{4} \partial_{x}^{4} f-\frac{1}{2}\left(\tau-\frac{1}{3}\right) h^{2} \partial_{x} f+\frac{1}{2} E_{\mathrm{b}} \tau h \partial_{x} \mathcal{H}[f]+\frac{p}{2 \rho g}=0 .
$$

Equations (103) and (104) are solved numerically by a finite difference scheme similar to that used in [4]. This leads to a system of nonlinear algebraic equations which is solved by Newton's method. We chose

$p(x)=\tilde{\epsilon} \mathrm{e}^{-x^{2} / 2}$.

We present computations for $\tilde{\epsilon}=0.05$. 


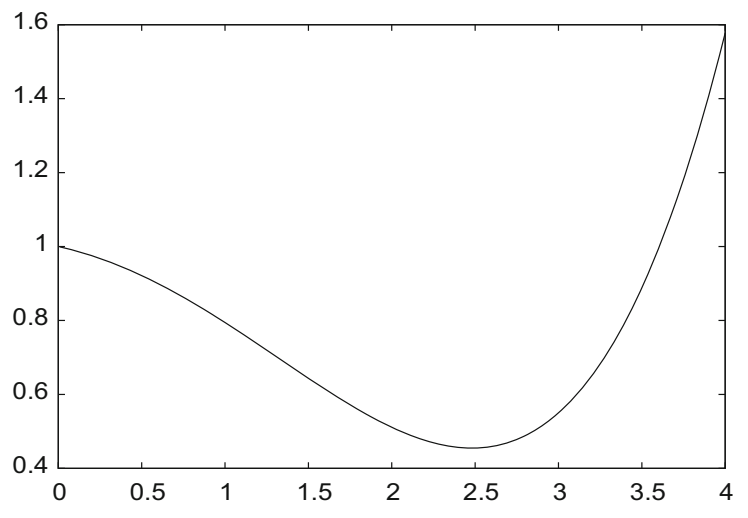

Fig. 5 Values of $F$ versus $k h$ predicted by (98) for $E_{\mathrm{b}}=2$ and $\tau=0.1$. The minimum value of $F$ is $F_{\min } \approx 0.455$

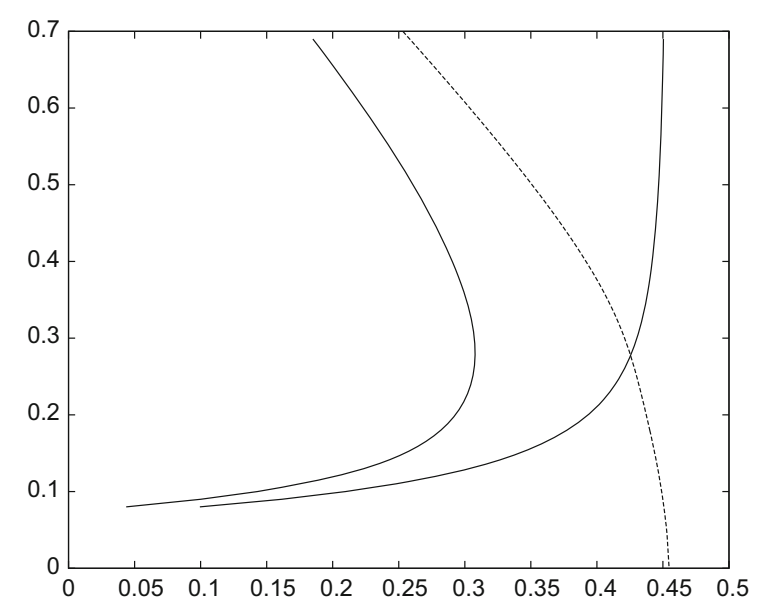

Fig. 6 Values of $\gamma$ versus $F$ for $\tau=0.1$ and $E_{\mathrm{b}}=2$. The solid curve further on the right corresponding to the linear model (104) has a vertical asymptote at $F \approx 0.455$. The solid curve further on the left has a turning point and corresponds to the weakly nonlinear model (103). The broken line bifurcates from the horizontal axis at $F \approx 0.455$ and corresponds to solitary waves with decaying tails

Numerical values of the maximum value $\gamma$ of $|f(x)|$ versus $F$ are presented in Fig. 6. Here $\gamma$ measures the maximum displacement of the free surface. It is similar to the quantity $|\eta(0)|$ of Fig. 4. We chose the same values of $E_{\mathrm{b}}=2$ and $\tau=0.1$ as in Fig. 5. The solid curve further on the right corresponds to the linear approximation (104). It has a vertical asymptote at $F \approx 0.455$. This value corresponds to the minimum $F_{\min }$ of Fig. 5 . This behaviour is qualitatively similar to that found in Sect. 3. It shows that that the linear approximation (104) becomes invalid as $F \rightarrow F_{\min }$.

We now show that this nonuniformity is removed by using the weakly nonlinear approximation (103). This is illustrated by the solid curve further on the left in Fig. 6. This curve was calculated by using the weakly nonlinear approximation (103). There is no longer a vertical asymptote and the curve is bent. This implies that there are two solutions for some values of $F$. The solution corresponding to the smaller value of $\gamma$ is a perturbation of a uniform stream, whereas that corresponding to the larger value of $\gamma$ is a perturbation of a solitary wave with decaying tail. To show this, we use a continuation method. The idea is to use solutions on the bent curve of Fig. 6 as an initial guess in the Newton iterations to compute solutions for a value of $\tilde{\epsilon}$ slightly smaller than 0.05 . The solutions obtained are then used as initial guesses to compute solutions for smaller values of $\tilde{\epsilon}$, and the procedure is repeated until solutions corresponding to $\tilde{\epsilon}=0$ are obtained (i.e. solutions without the distribution of pressure). The solution obtained by starting with an initial guess on the lower part of the bent curve of Fig. 6 is $f(x)=0$ (i.e. a uniform stream). The solution obtained by starting with an initial guess on the upper part of the bent curve of Fig. 6 is a solitary wave with decaying tails. Such solutions form a branch which bifurcates from the horizontal axis $\gamma=0$ at $F=F_{\min }$. It is shown by the broken line in Fig. 6 . Solitary waves with decaying tails have profiles qualitatively similar to that of Fig. 3. Typical profiles are shown in Figs. 7 and 8. As $\gamma \rightarrow 0$, more and more oscillations appear on the free surface. Such waves have been calculated before in the absence of electric field (see [9] and also [3] for a review and further references). 


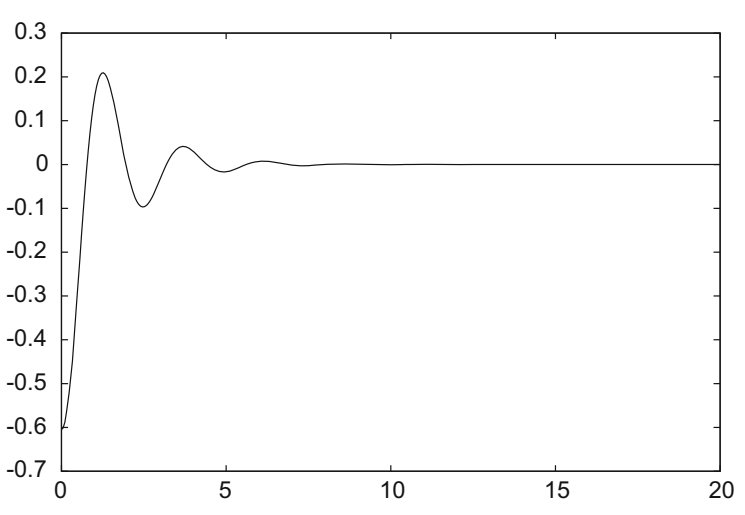

Fig. 7 Free surface profile of a solitary wave for $\tau=0.1, E_{\mathrm{b}}=2$ and $\gamma=0.6$. Only half of the profile is shown

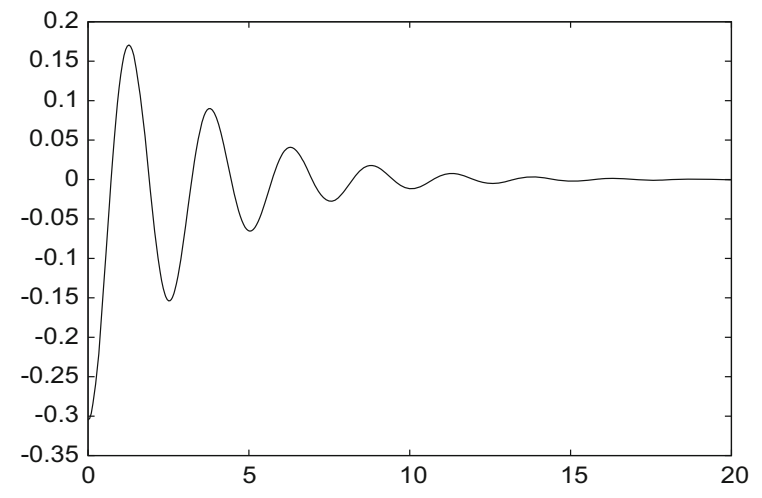

Fig. 8 Free surface profile of a solitary wave for $\tau=0.1, E_{\mathrm{b}}=2$ and $\gamma=0.3$. Only half of the profile is shown

\section{Conclusions}

We have considered the problem of free surface flows generated by a moving distribution of pressure in the presence of electric field. We have first developed a linear theory by assuming that the magnitude of the pressure is small. The results show that the displacement of the free surface is unbounded as the Froude number approaches a critical value corresponding to the minimum in the linear dispersion relation. We showed how to remove this uniformity by developing a weakly nonlinear theory. These findings generalise previous investigations in the absence of electric field.

Acknowledgments This work was supported in part by EPSRC under grant EP/J019569/1.

Open Access This article is distributed under the terms of the Creative Commons Attribution License which permits any use, distribution, and reproduction in any medium, provided the original author(s) and the source are credited.

\section{References}

1. Forbes LK, Schwartz LW (1982) Free surface flow over a semi-circular obstruction. J Fluid Mech 114:299-314

2. Parau E, Vanden-Broeck J-M, Cooker M (2005) Nonlinear three dimensional gravity capillary solitary waves. J Fluid Mech 536:99105

3. Vanden-Broeck J-M (2010) Gravity-capillary free-surface flows. Cambridge University Press, Cambridge

4. Papageorgiou DT, Vanden-Broeck J-M (2004) Large amplitude capillary waves in electrified fluid sheets. J Fluid Mech 508:71-88

5. Gleeson H, Hammerton P, Papageorgiou DT, Vanden-Broeck J-M (2007) A new application of the Korteweg-de Vries Benjamin-Ono equation in interfacial electrohydrodynamics. Phys Fluids 19:031703

6. Melcher JR, Taylor GI (1969) Electrohydrodynamics: a review of the role of interfacial shear stresses. Annu Rev Fluid Mech 1:111-146

7. Griffing EM, Bankoff SG, Miksis MJ, Schluter RA (2006) Electrohydrodynamics of thin flowing films. ASME Trans J Fluids Eng $128: 276-283$

8. Hunt MJ (2013) Linear and nonlinear free surface flows in electrohydrodynamics. PhD thesis, University College London, London

9. Vanden-Broeck J-M, Dias F (1992) Gravity-capillary solitary waves in water of infinite depth and related free-surface flows. J Fluid Mech 240:549-557 